\title{
Investigation and identification of let-7a related functional proteins in gastric carcinoma by proteomics
}

\author{
Yimin Zhu ${ }^{\mathrm{a}}$, Xingyuan Xiao ${ }^{\mathrm{b}}$, Lairong Dong ${ }^{\mathrm{a}}$ and Zhiming $\mathrm{Liu}^{\mathrm{c}, *}$ \\ ${ }^{a}$ Department of Oncology, The Second Hospital of Jiaxing, Jiaxing, China \\ ${ }^{\mathrm{b}}$ Department of General Surgery, The First People's Hospital of Kunshan, Kunshan, China \\ ${ }^{\mathrm{c}}$ Gastrointestine and Gland Surgery Division, The First Affiliated Hospital of Guangxi Medical University, \\ Nanning, China
}

Received: January 7, 2012

Accepted: April 25, 2012

\begin{abstract}
MicroRNAs are small noncoding RNA molecules that control expression of target genes. Our previous studies show that let-7a decreased in gastric carcinoma and that up-regulation of let-7a by gene augmentation inhibited gastric carcinoma cell growth both in vitro and in vivo, whereas it remains largely unclear as to how let-7a affects tumor growth. In this study, proteins associated with the function of let-7a were detected by high throughout screening. The cell line of SGC-7901 stablely overexpressing let-7a was successfully established by gene cloning. Two-dimensional gel electrophoresis (2-DEy was used to separate the total proteins of SGC-7901/let-7a, SGC-7901/EV and SGC-7901, and PDQuest software was applied to analyze 2-DE images. Ten different protein spots were identified by MALDI-TOF-MS, and they may be the proteins associated with let-7a function. The overexpressed proteins included Antioxidant protein 2, Insulin-like growth factor binding protein 2, Protein disulfide isomerase A2, C-1-tetrahydrofolate synthase, Cyclin-dependent kinase inhibitor1 (CDKN1) and Rho-GTPase activating protein 4 . The underexpressed proteins consisted of S-phase kinase-associated protein 2 (Spk2), Platelet membrane glycoprotein, Fibronectin and Cks1 protein. Furthermore, the different expression levels of the partial proteins (CDKN1, Spk2 and Fibronectin) were confirmed by western blot analysis. The data suggest that these differential proteins are involved in a novel let-7a signal pathway and these findings provide the basis to investigate the functional mechanisms of let-7a in gastric carcinoma.
\end{abstract}

Keywords: Proteomics, miRNA, gastric carcinoma

\section{Introduction}

MicroRNAs (miRNAs) are a class of naturally occurring small noncoding RNAs that regulate gene expression by targeting mRNAs for translational repression or cleavage $[1,2]$. They bind through par-

\footnotetext{
${ }^{*}$ Corresponding author: Zhiming Liu, Gastrointestine and Gland Surgery Division, The First Affiliated Hospital of Guangxi Medical University, 6th Shuangyong Road, Nanning 530021, P.R. China. Tel.: +86771 5356738; Fax: +86771 5356738; E-mail: zym330422@163.com.
}

tial sequence homology to the $3^{\prime}$-untranslated region $\left(3^{\prime}\right.$-UTR) of target genes. Because of this unique feature, a single miRNA has multiple targets. Thus, miRNAs could regulate a large fraction of proteincoding genes, and as high as $30 \%$ of all genes could be miRNA targets [3]. As a new layer of gene regulation mechanism, miRNAs have diverse functions, including the regulation of cellular differentiation, proliferation, and apoptosis [4, 5]. However, although miRNAs have been the subject of extensive research in recent years, the molecular basis of miRNA-mediated 
gene regulation and the effect of these genes on tumor growth remain largely unknown because of our limited understanding of miRNA-target genes. Evidence suggests that perfect seed pairing may not necessarily be a reliable predictor for miRNA-target interactions [6], which may explain why many predicted target sites are nonfunctional. A recent study also suggests that there may be at least three types of miRNA-mRNA interactions in mammals [7]. Hence, with few exceptions, a large portion of the physiologic targets for miRNAs remain to be identified or verified experimentally.

Gastric carcinoma is the second most prevalent cancer and there are still 0.3 million deaths from gastric carcinoma annually in China. One particular microRNA, let-7, has been reported as a novel candidate of tumor suppressor genes involved several cancers. In our previous study, it was found that reduced let-7a was one of the earliest biochemical events associated with the progression of gastric mucosa cancerization [8]. Therefore, we then conducted a study to determine the influence of let-7a overexpression on cell proliferation or the cell cycle in an in vitro and in vivo cell model for gastric carcinoma. The results demonstrated that a lentiviral vector was capable of upregulating let-7a, resulting in impressive anticancer effects $[9,10]$. Based on our results, overexpression of the let-7a gene provides a potential therapeutic approach for the management of gastric carcinoma. Our studies strongly support the notion that let-7 functions as a tumor suppressor miRNA in gastric carcinoma while the molecular mechanism used by let-7 to repress tumor formation is largely unknown.

Proteomics is an effective platform to globally detect and characterize proteins. Comparative proteomic approach is the main strategy of proteomics to analyze and compare the differentially expressed proteins $[11,12]$. On the other hand, gene augmentation is an important method in treating cancers. Thus, we used gene cloning to overexpress let-7a in the human gastric carcinoma cell line SGC-7901 which was named SGC-7901/let-7a. SGC-7901 cells transduced with empty lentiviral vectors were used as the negative control group, which was named SGC7901/EV. The parental SGC-7901 cells were used as a blank control group. A comparative proteomic approach was performed to identify the differential proteins among SGC-7901/let-7a and control cells. In our study, we used two-dimensional gel electrophoresis (2-DE) to detect the differentially expressed proteins in SGC-7901/let-7a and control cell lines. The differentially expressed proteins among the three cell lines were identified by matrix-assisted laser desorption/ionization time-of-flight mass spectrometry (MALDI-TOF-MS). Furthermore, the relationships of partial identified differential proteins with let-7a in SGC-7901 were studied by Western blot analysis. The results presented here will provide clues for further study of functional mechanism of let-7a in treating human gastric carcinomas.

\section{Materials and methods}

\subsection{Cell culture}

Human gastric carcinoma cells SGC-7901, and human embryonic kidney (HEK) 293T cells were cultured in Dulbecco's modified Eagle's medium (DMEM, Gibco) with $10 \%$ heat-inactivated FBS, $2 \mathrm{mML}$-glutamine, $100 \mathrm{U} / \mathrm{ml}$ penicillin and $100 \mu \mathrm{g} / \mathrm{ml}$ streptomycin at $37^{\circ} \mathrm{C}$ in a humidified atmosphere of $5 \% \mathrm{CO}_{2}$.

\subsection{Plasmid construction}

The DNA sequence of let-7a was obtained from Gene Bank (ID No. NM_0062). The primers (forward primer, 5'-AGGATCC AAAGGTGGTGGTAAGA GGGTGAT3'; reverse primer, 5'-AGTCGAC ATA AGACAAGAAGCAAAAGGTTT-3') were designed to amplify the entire coding sequence of let-7a and produced an amplified product of $600 \mathrm{bp}$. The rounds of PCR were performed according to the TaKaRa LA Taq data sheet. After PCR, $2 \mu l$ of the amplified product was electrophoresed in $1 \%$ agarose gelatin gels buffered with $1 \times$ TBE. The plasmid of Pwpxl-MOD2, a gift of Dr. Liu (Shanghai Jiaotong University), was used to construct the let-7a expression vector. The PCR product and Pwpxl-MOD2 were digested with BamHI and SalI. The fragment was isolated from agarose gel and ligated into the cut vector to form Pwpxl-MOD2let-7a, which was then transfected into $293 \mathrm{~T}$ cells. While the negative control plasmid Pwpxl-MOD2 just was not digested and ligated any oligonucleotide. The transfer plasmid Pwpxl-MOD2-let-7a expressing let-7a shRNA and the control negative plasmid Pwpxl-MOD2 were constructed for packaging lentiviral vectors. Both of them can express enhanced green fluorescent protein (EGFP). 


\subsection{Generation and titer of lentivirus}

Lentiviral vector supernatant was produced by fourplasmid cotransfection in the HEK 293T transient system. Briefly, $20 \mu \mathrm{g}$ Pwpxl-MOD2-let-7a (transfer plasmid) and $15 \mu \mathrm{g}$ pMDlg-Prre, $10 \mu \mathrm{g}$ pRsv-REV, pMD2 $\mathrm{G} 7.5 \mu \mathrm{g}$ (packaging plasmids)were used to cotransfect HEK 293T cells using calcium phosphate co-precipitation. $3.0 \times 10^{6} 293 \mathrm{~T}$ cells were seeded in $10-\mathrm{cm}$ cell culture dishes, and allowed to reach a confluence of $70-80 \%$ for transfection. Culture medium was replaced $16-18 \mathrm{~h}$ after transfection with $15 \mathrm{ml}$ of $10 \%$ FCS DMEM per dish. Viral supernatant was harvested $72 \mathrm{~h}$ after transfection and filtered through a $0.45-\mu \mathrm{m}$ cellulose acetate filter, then ultracentrifuged at $50,000 \mathrm{~g}$ at $4^{\circ} \mathrm{C}$ for $2 \mathrm{~h}$. The viral pellet was resuspended in serum-free DMEM, divided into aliquots and frozen at $-70^{\circ} \mathrm{C}$. The control lentiviral vector supernatant was made similarly. The functional titer of virus in SGC-7901 cells was determined by in vitro transduction and flow cytometry(FCM) analysis [13].

\subsection{Establishment of stable overexpression lines}

SGC-7901 cells were transduced with specific or empty lentiviral vectors using a multiplicity of infection of 5 and selected for stable integrants using EGFP reporter gene. Briefly, the transduced SGC-7901 cells were maintained in culture medium for four to five passages until EGFP expression was stable; the transduced cells were trypsinized, diluted to 10 cells $/ \mathrm{ml}$, and $100 \mu \mathrm{l}$ of cells were seeded in each well of a 96-well plate. After 10 days of incubation, the single colony strongly expressing EGFP was selected under a fluorescent microscopy. The EGFP-positive cells were named SGC-7901/let-7a, SGC-7901/EV, respectively. Passages 10-20 were used in the experiments. There was no significant change in morphological characterization from passage 10 to 20 . Subsequently, more than $98 \%$ transduced cells were found to strongly express EGFP using fluorescent microscopy (Fig. 1). Those cells expressing EGFP were selected through FCM as stable overexpression lines.

\subsection{Real-time RT-PCR analysis of let-7a}

Total RNA was isolated from cultured cells using Trizol reagent (Invitrogen). Let-7a was quantified with real-time RT-PCR. Primer sequences were designed using the Primer Express software program (PE Applied Biosystems): forward primer: GCCGCTGAGGTAGTAGGTTGTA; reverse primer: GTGCAGGGTCCGAGGT. 18sRNA was used as an endogenous control. The reaction was performed in a volume of $25 \mu \mathrm{l}$ containing oligonucleotide primers ( $2 \mu \mathrm{l}$ each), cDNA template $(0.5 \mu \mathrm{l})$ and SYBR Green $\operatorname{mix}(25 \mu \mathrm{l})$ in duplicate. The amplifications were performed in the ABI Prism 7000 Sequence Analyzer using the following cycle program: $95^{\circ} \mathrm{C}$ for $5 \mathrm{~min}$, $95^{\circ} \mathrm{C}$ for $20 \mathrm{~s}, 50^{\circ} \mathrm{C}$ for $15 \mathrm{~s}, 68^{\circ} \mathrm{C}$ for $20 \mathrm{~s}$ followed by 40 cycles and then $68^{\circ} \mathrm{C}$ for $10 \mathrm{~min}$. The relative expression level of the let-7a gene was calculated by comparing the threshold cycle $(\mathrm{Ct})$ values of samples to that of the reference (SGC-7901). The Ct values for each reaction were determined using Taq-Man SDS analysis software. For each sample tested, the duplicate $\mathrm{Ct}$ values were averaged, Accordingly, ${ }^{\Delta} \mathrm{Ct}=($ mean of $\mathrm{SGC}-7901 /$ let-7a Ct) - (the mean of 18sRA Ct), and ${ }^{\Delta \Delta} \mathrm{Ct}={ }^{\Delta} \mathrm{Ct}(\mathrm{SGC}-7901 /$ let $-7 a)-{ }^{\Delta} \mathrm{Ct}$ (SGC-7901). The relative gene expression in a particular sample was then given by the relative amount of the target: $2^{-\Delta \Delta \mathrm{Ct}}$ value [14] (Fig. 2).

\section{6. $2-D E$}

Monolayer cultures of three cell lines SGC-7901/let7a, SGC-7901/EV, and SGC-7901 were harvested, and lysed in lysis buffer (7 M urea, $2 \mathrm{M}$ thiourea, $100 \mathrm{mM}$ DTT, $4 \%$ Chaps, $40 \mathrm{mM}$ Tris, $2 \%$ Pharmalyte, $1 \mathrm{mg} / \mathrm{ml}$ DNase I). After being incubated at $37^{\circ} \mathrm{C}$ for $1 \mathrm{~h}$, the lysates were centrifuged at $15000 \mathrm{rpm}$ for $30 \mathrm{~min}$ at $4{ }^{\circ} \mathrm{C}$. The supernatant was transferred and the concentration of the total proteins was determined using 2D Quantification kit (Amersham Biosciences). Triplicate gels were made for each cell line and a total amount of $1.0 \mathrm{mg}$ of protein per gel was analyzed. Protein samples were diluted to $450 \mu \mathrm{l}$ with rehydration solution [7 M urea, $2 \mathrm{M}$ thiourea, 0.2\% DTT, 0.5\% (v/v) Ph 4-7 IPG buffer (Amersham Biosciences), and trace bromophenol blue] and applied to IPG strips (pH 4-7 L, $240 \mathrm{~mm} \times 3 \mathrm{~mm} \times 0.5 \mathrm{~mm}$, Amersham Biosci-ences) by $24 \mathrm{~h}$ rehydration at $30 \mathrm{~V}$. Then proteins were focused successively for $1 \mathrm{~h}$ at $500 \mathrm{~V}, 1 \mathrm{~h}$ at $1000 \mathrm{~V}$ and $8.5 \mathrm{~h}$ at $8000 \mathrm{~V}$ to give a total of $68 \mathrm{kVh}$ on an IPGphor (Amersham Biosciences). Focused IPG strips were equilibrated for $15 \mathrm{~min}$ in a solution (6 M urea, $2 \%$ SDS, $30 \%$ glycerol, $50 \mathrm{mM}$ Tris- $\mathrm{HCl}, \mathrm{pH} 8.8$ and $1 \%$ DTT), and then for an additional $15 \mathrm{~min}$ in the same 

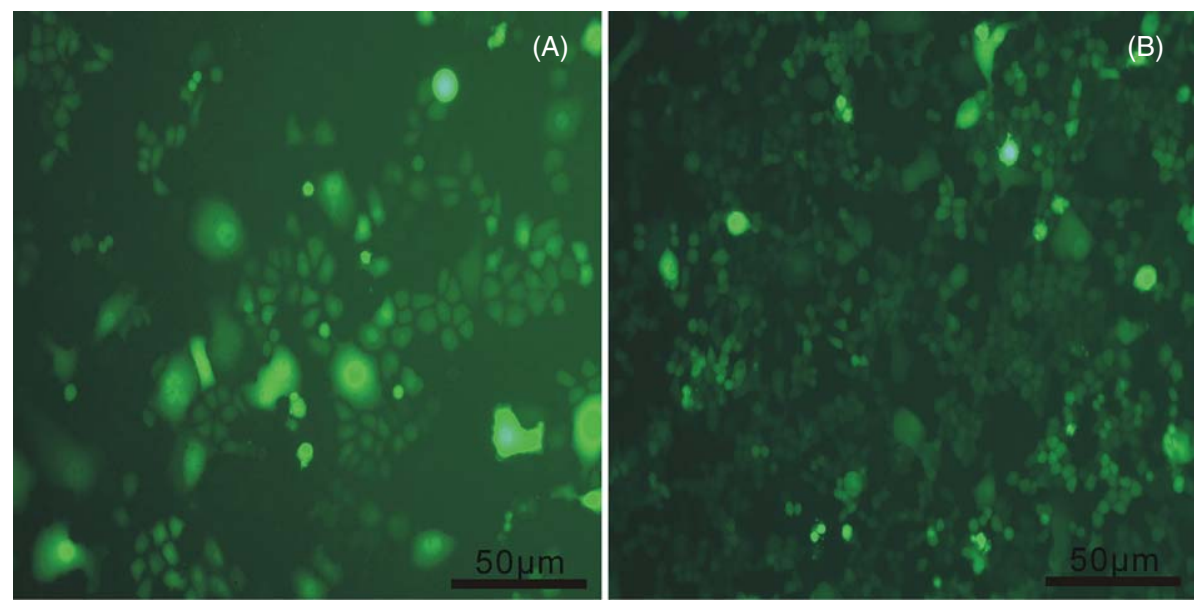

Fig. 1. SGC-7901 transduced with lentiviral vectors. (A) Selected cells (parental SGC-7901) were transduced with let-7a lentiviral vectors at a multiplicity of infection of 5 and selected for stable integrants using EGFP reporter gene. Original magnification: $\times 200$. (B) Selected cells (parental SGC-7901) were transduced with empty lentiviral vectors at a multiplicity of infection of 5 and selected for stable integrants using EGFP reporter gene. Calibration bar is $50 \mu \mathrm{m}$ for both panels.

(A)

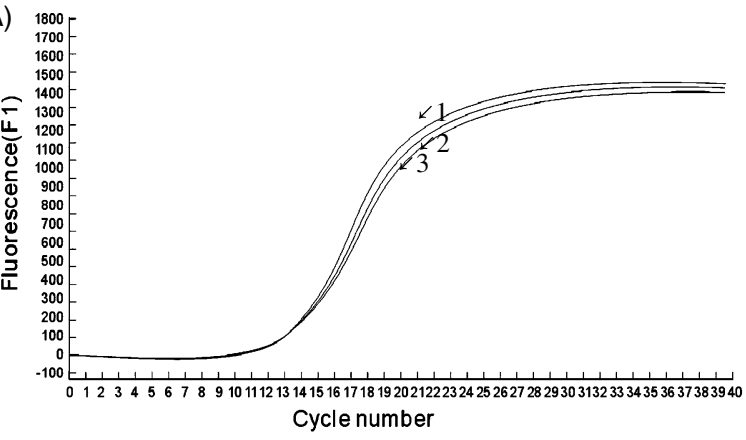

(C)

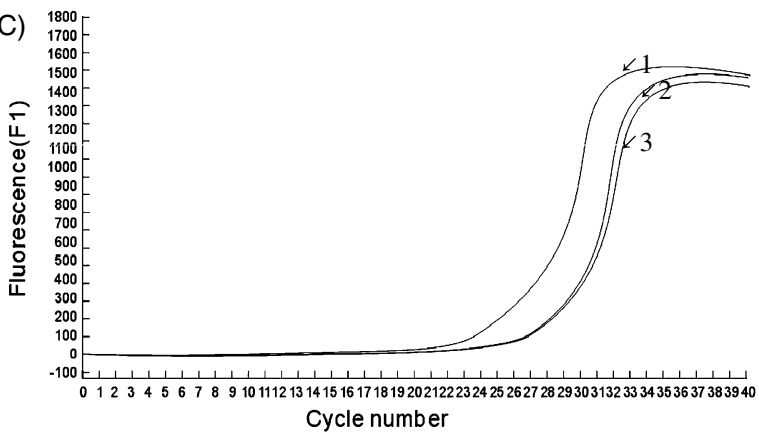

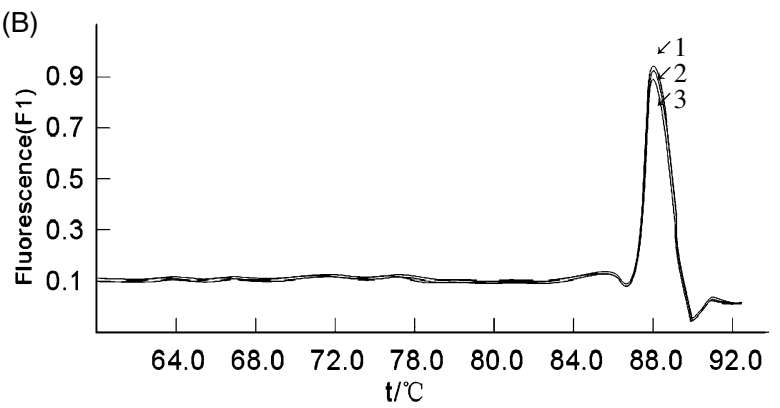

(D)

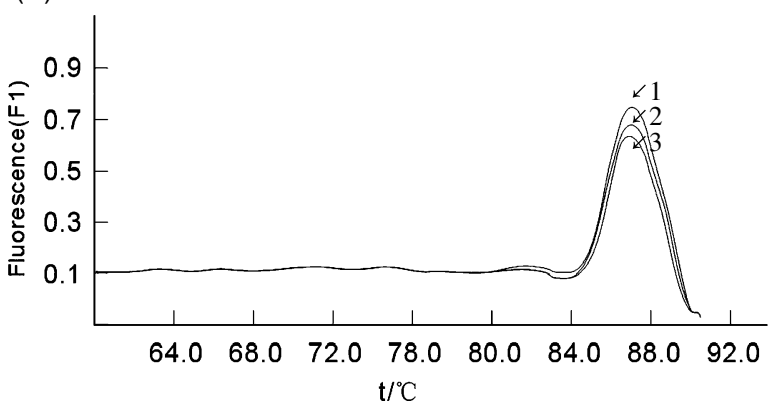

Fig. 2. Representative real-time quantitative RT-PCR analysis for of let-7a level. The expression level of let-7a was examined and was normalized to that of 18 S RNA. (A) Graph shows that 18 S RNA among SGC-7901/let-7a, SGC-7901/EV and SGC-7901 cell lines has very little difference. (C) Graph showed that let-7a significantly increased in the SGC-7901/let-7acell line. (B) and (D) Graph showed the melt curve of representative PCR products of 18 S RNA and let-7a respectively. 1: SGC-7901/let-7a; 2:SGC-7901/EV; 3: SGC-7901. 
solution except that DTT was replaced by $2.5 \%$ iodoacetamide (Sigma-Aldrich). After equilibration, seconddimension sodium dodecyl sulfate-polyacrylamide gel electrophoresis (SDS-PAGE) was performed on the Ettan DALT II system (Amersham Biosciences). After SDS-PAGE, the Blue Silver staining method, a modified Neuhoff's colloidal coomassie blue G-250 stain (Amersham Biosciences), was used to visualize the protein spots in the 2-DE gels [15].

\subsection{Gel scanning and image analysis}

The stained 2-DE gels were scanned with MagicScan software on Imagescanner (maximum resolution $9600 \times 9600$ dpi) (Amersham Biosciences), and analyzed using PDQuest system (Bio-Rad Laboratories, Hercules, CA, USA) according to the protocols provided by the manufacturer. To minimize the contribution of experimental variations, three separate gels were prepared for each cell line. The gel spot pattern of each gel was summarized in a standard after spot matching. Thus, we obtained one standard gel for each cell line. Spot intensities were quantified by calculation of spot volume after normalization of the image using the total spot volume normalization method multiplied by the total area of all the spots. The change index was defined as the ratio between the spot percentage relative volumes in SGC-7901/let-7a, SGC-7901/EV, and SGC-7901 cell lines. All indexes were calculated based on the mean of the spot intensities of all nine gels, with standard deviations. Proteins were classified as being differentially expressed between the two cell lines when spot intensity showed a difference $\geq 5$-fold variation in SGC-7901/let-7a, SGC-7901/EV in comparison to SGC-7901. Significant differences in protein expression levels were determined by Student's $t$-test with a set value of $P<0.05$.

\subsection{Protein identification by MALDI-TOF-MS}

Selected differential protein spots were excised from preparative gels using punch and in-gel digestion was performed. The gel-spots were washed three times with two-distillation water and destained with fresh solution containing $100 \mathrm{mM} \mathrm{NH}_{4} \mathrm{HCO}_{3}$ in $50 \%$ acetonitrile at $37^{\circ} \mathrm{C}$. After drying in a vacuum centrifuge, the gel-pieces were incubated in $10 \mu$ l digestion solution consisted of $40 \mathrm{mM} \mathrm{NH}_{4} \mathrm{HCO}_{3}$ in $9 \%$ acetonitrile solution, and $20 \mu \mathrm{g} / \mathrm{ml}$ proteomics grade trypsin (Promega,
USA) for $10-12 \mathrm{~h}$ at $37^{\circ} \mathrm{C}$. The tryptic peptide mixture was extracted and purified with a Millipore ZipTip C18 column (Millipor, Bedford, MA, USA). The purified tryptic peptide mixture was mixed with containing $\alpha$-cyano-4-hydroxycinnamic (CCA) (Sigma-Aldrich Co.) matrix solution and vortexed gently. A volume $(1 \mu l)$ of the mixture CCA matrix was loaded on a stainless steel plate and air-dried, and then was analyzed with Voyager System DE-STR 4307 MALDI-TOF Mass Spectrometer (AppliedBiosystems, Foster City, CA, USA). The standard peptide mixture was spotted at the same time to correct the machine. In peptide mass fingerprint (PMF) map database searching, Mascot Distiller was used to get the monoisotopic peak list from the raw mass spectrometry files. Peptide matching and protein searches against the Swiss-Prot databases were performed using the Mascot search engine (http://www.matrixscience.com/) with a mass tolerance of $\pm 50 \mathrm{ppm}$.

\subsection{Western blot analysis of let-7a related proteins}

The cells were harvested from flasks, and lysed in a lysis buffer $(50 \mathrm{mM}$ Tris, $\mathrm{pH} 7.4,100 \mathrm{mM} \mathrm{NaCl}$, $1 \mathrm{mM} \mathrm{MgCl}_{2}, 2.5 \mathrm{mM} \mathrm{Na}_{3} \mathrm{VO}_{4}, 1 \mathrm{mM}$ PMSF, $2.5 \mathrm{mM}$ EDTA, $0.5 \%$ Triton X-100, 0.5\% NP-40, $51 \mathrm{~g} / \mathrm{mL}$ of aprotinin, pepstatin $\mathrm{A}$, and leupeptin) for $60 \mathrm{~min}$ on ice, followed by centrifuging at $11000 \times \mathrm{g}$ for $15 \mathrm{~min}$ at $4{ }^{\circ} \mathrm{C}$ to remove cell debris. The concentration of proteins was determined using Bradford reagent (BioRad Laboratories). Protein samples $(500 \mu \mathrm{g})$ were separated on $12.5 \%$ SDS-PAGE gels, and transferred to Hybond-P PVDF membranes (Amersham Biosciences, Stockholm, Sweden). After blocking with 5\% non-fat dry milk in TBS-T buffer (20 mM Tris, pH 7.6, $100 \mathrm{mM} \mathrm{NaCl}, 0.1 \%$ Tween-20) for $2 \mathrm{~h}$ at room temperature, the membranes were respectively probed with $1: 1000$ dilution of anti-CDKN1, anti-Skp2 and antiFibronectin (Santa Cruz Biotechnology, CA, USA) overnight at $4{ }^{\circ} \mathrm{C}$, and probed with $1: 1000$ dilution of anti-GAPDH (Sigma-Aldrich) to correct for differences in protein loading, followed by incubation in a 1:2000 dilution of secondary antibodies conjugated to horseradish peroxidase (Amersham Biosciences) for $1 \mathrm{~h}$ at room temperature. Protein bands were detected using ECL detection system (Amersham Biosciences). All of the western immunoblots were performed at least three times. 


\section{Results}

\subsection{Overexpression of let-7a in gastric carcinoma cells transduced with let-7a lentiviral vector}

The lentiviral-vector-overexpression let-7a gene and control empty vectors were constructed and used to transduce the gastric carcinoma cell line (SGC-7901). Colonies with strong EGFP expression were selected to construct let-7a stable overexpressing cells (Fig. 1). By in vitro transduction and flow cytometry (FCM) analysis, results showed that the functional titer of virus was $5.0 \times 10^{8} \mathrm{TU} / \mathrm{ml}$. In order to verify the let- $7 \mathrm{a}$ overexpressing effects, total RNAs were isolated from SGC-7901, SGC-7901/EV and SGC-7901/let-7a cells. We characterized let-7a at the RNA levels by real-time RT-PCR (Fig. 2). The copies of let-7a in SGC-7901/ let-7a cells were 9-fold higher than in SGC-7901 cells, SGC-7901/EV cells, respectively $(P<0.001)$. There was no significant increase in empty vector-transduced cells, compared to the parental cells (Fig. 3). The overexpression of let-7a with lentiviral vectors was significant and specific. Lentiviral constructs can increase the expression of let-7a gene efficiently.

\subsection{2-D gel analysis of differentially expressed proteins}

To identify the proteins associated with the function of let-7a in SGC-7901, we initiated a comparative

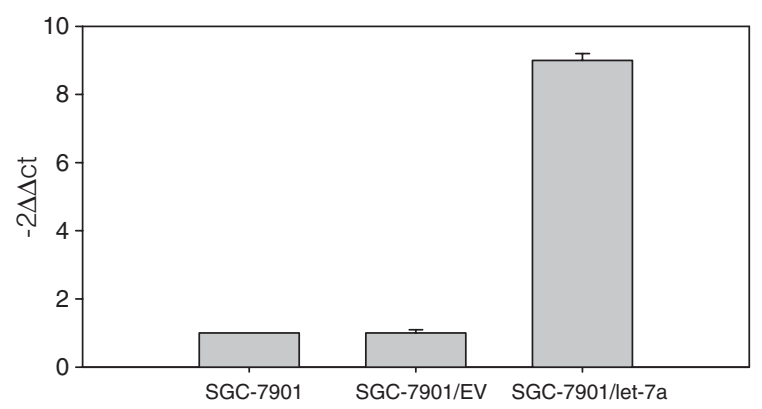

Fig. 3. Real-Time RT-PCR analysis of let-7a in SGC-7901, SGC7901/EV and SGC-7901/let-7a cells. The relative expression level of the let-7a was calculated by comparing the threshold cycle $(\mathrm{Ct})$ values of samples to that of the reference (SGC-7901). For each sample tested, the three times of duplicate $\mathrm{Ct}$ values were averaged, The copies of let-7a in SGC-7901/let-7a cells were 9-fold higher than in SGC-7901cells and SGC-7901/EV cells, respectively $(P=0.00)$. There was no significant increase in SGC-7901/EV cells, comparing to SGC-7901 cells. proteomic study of SGC-7901/let-7a, SGC-7901/EV, and SGC-7901 cell lines. In the preliminary experiment, 2-DE maps of SGC-7901/let-7a, SGC-7901/EV, and SGC-7901 were performed for several repeated runs. We found that most of the differentially expressed proteins were included in the $\mathrm{pH}$ range from 3 to 10. To isolate these proteins more efficiently, the narrow $\mathrm{pH}$ 3-10 IPG strips for the first dimension were used to better separate the total proteins. In the $\mathrm{pH}$ range 3-10, three representative 2-DE maps from SGC-7901/let-7a, SGC-7901/EV, and SGC-7901 are shown in Fig. 4A. For a reliable analysis of protein expression, 2-DE of each cell line was performed in triplicate. The 2-DE patterns of each cell line were highly reproducible and well-resolved. The 2DE maps of SGC-7901/let-7a, SGC-7901/EV, and SGC-7901 displayed about 1026, 1046 and 1043 protein spots respectively. Maps of SGC-7901/EV and SGC-7901 were similar to each other, while the map of SGC-7901/let-7a was distinguishable from SGC7901/EV and SGC-7901. Close-ups of the region of the gels showing differential expression proteins between SGC-7901/let-7a, SGC-7901/EV, and SGC-7901 are shown in Fig. 4B. Image analysis revealed that 6 spots (1-6) were specifically upregulated $(\geq 5$-fold upregulated; $P<0.05)$ in the SGC-7901/let-7a while 4 spots (7-10) were decreased ( $\geq 5$-fold downregulated; $P<0: 05)$ in comparison with the expression in SGC-7901/EV and SGC-7901.

\subsection{Identification of differential expression proteins by MALDI-TOF-MS}

The 10 differentially expressed protein spots, marked with the numbers and arrows in Fig. 4A, were excised from coomassie brilliant blue-stained gels, in situ digested with trypsin and analyzed by MALDI-TOF-MS. High-quality PMFs were obtained, and all the 10 differentially expressed protein were identified. The MALDI-TOF mass spectrometry maps of spots 5, 7, 9 are shown in Fig. 5A, B and C, respectively. Their monoisotopic peaks were input into the Mascot search engine to search the Swiss-Prot database and the query results showed that protein spots were Antioxidant protein 2, Insulin-like growth factor binding protein 2, Protein disulfide isomerase A2, C-1-tetrahydrofolate synthase, Cyclin-dependent kinase inhibitor1, Rho-GTPase activating protein 4, S-phase kinase-associated protein 2, Platelet membrane glycoprotein, Fibronectin and Cks1 protein. The 

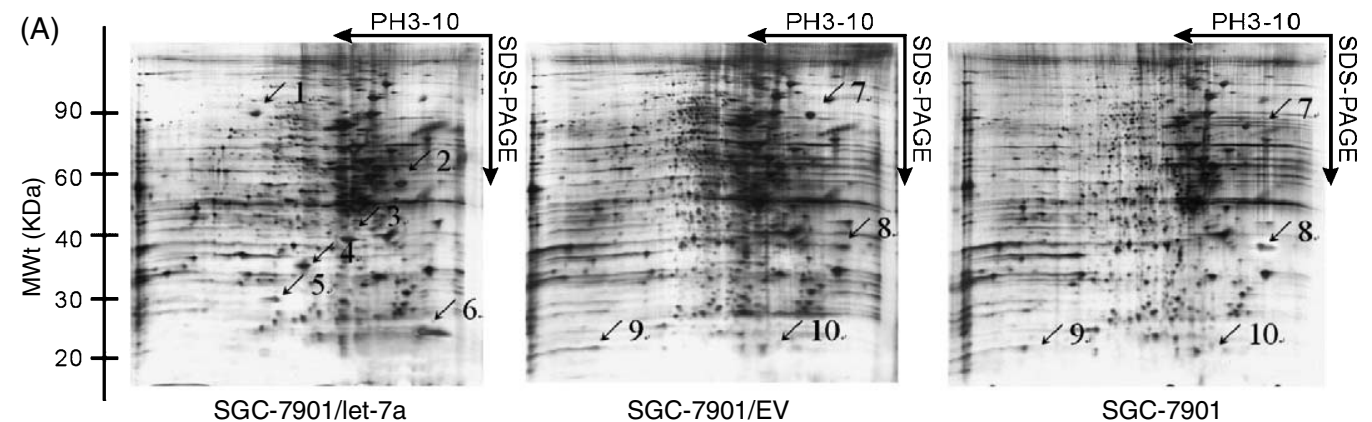

(B)

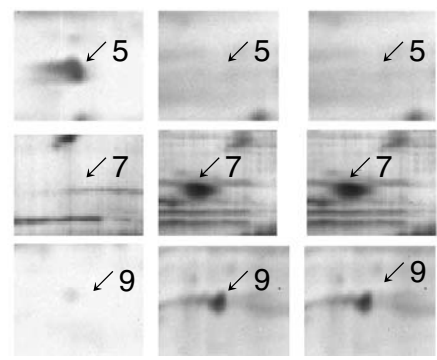

Fig. 4. Representative 2-DE maps of SGC-7901, SGC-7901/EV and SGC-7901/let-7a cell lines. (A) 10 differential expression proteins spotsmarked with arrows were identified using 2-DE. (B) Close-up image of partial differential expression protein spots among SGC-7901/let-7a, SGC-7901/EV and SGC-7901 cells.

Table 1

MALDI-TOF-MS identified proteins in SGC-7901/let-7a vs. SGC-7901 and SGC-7901/EV

\begin{tabular}{|c|c|c|c|c|c|c|c|}
\hline Spot no. & Protein name & $\begin{array}{c}\text { Accession } \\
\text { number }\end{array}$ & Matching & $\mathrm{pI}$ & $\begin{array}{c}\text { Molecular } \\
\text { weight }\end{array}$ & $\%$ Coverage & Classification \\
\hline 1 & Antioxidant protein 2 & P30041 & $7 / 19$ & 8.28 & 82904 & 40.9 & Metabolism \\
\hline 2 & Insulin-like growth factor binding protein 2 & P18065 & $7 / 13$ & 4.31 & 50137 & 42.1 & Signal transduction \\
\hline 3 & Protein disulfide isomerase A2 & Q13087 & $6 / 18$ & 6.04 & 39206 & 51.5 & Metabolism \\
\hline 4 & C-1-tetrahydrofolate synthase & P11586 & $7 / 23$ & 6.79 & 34428 & 65.6 & Metabolism \\
\hline 5 & Cyclin-dependent kinase inhibitor 1 & P38936 & $4 / 13$ & 7.24 & 31119 & 38.3 & Cell cycle regulatory \\
\hline 6 & Rho-GTPase activating protein 4 & P98171 & $9 / 22$ & 3.73 & 27055 & 55.5 & Signal transduction \\
\hline 7 & S-phase kinase-associated protein 2 & Q13309 & $7 / 11$ & 3.97 & 77439 & 32.5 & Cell cycle regulatory \\
\hline 8 & Platelet membrane glycoprotein & P08514 & $5 / 12$ & 3.62 & 38107 & 47.1 & Signal transduction \\
\hline 9 & Fibronectin & P02751 & $8 / 15$ & 9.61 & 25933 & 52.6 & Signal transduction \\
\hline 10 & Cks1 protein & P61024 & $4 / 11$ & 5.13 & 26214 & 61.9 & Cell cycle regulatory \\
\hline
\end{tabular}

Differentially labelled proteins form 2D analysis were identified by MALDI-TOF peptide mass fingerprinting. The Swiss-Prot accession number, peptides matching, pI, molecular weight and \% coverage of analysed peptides are shown for each protein; Proteins displaying an average fold-difference of $\geq 5$ fold up (+) or down ( - ) regulation between pairs of conditions were considered as significant difference.

annotation of all the identified proteins is summarized in Table 1. Based on protein functions using information from the database of Swiss-Prot websites, 10 identified proteins associated with stable overexpression of let- 7 a were categorized into 3 protein groups. Most of the differentially expressed proteins were cell cycle regulators and proteins related to signal transduction or metabolism.

\subsection{Identification of let-7a related proteins by Western blot analysis}

To determine whether overexpressing let-7a in SGC-7901 has an effect on Cyclin-dependent kinase inhibitor 1, S-phase kinase-associated protein 2 and Fibronectin protein. Western blot analysis of the expression levels of these proteins was performed. The 

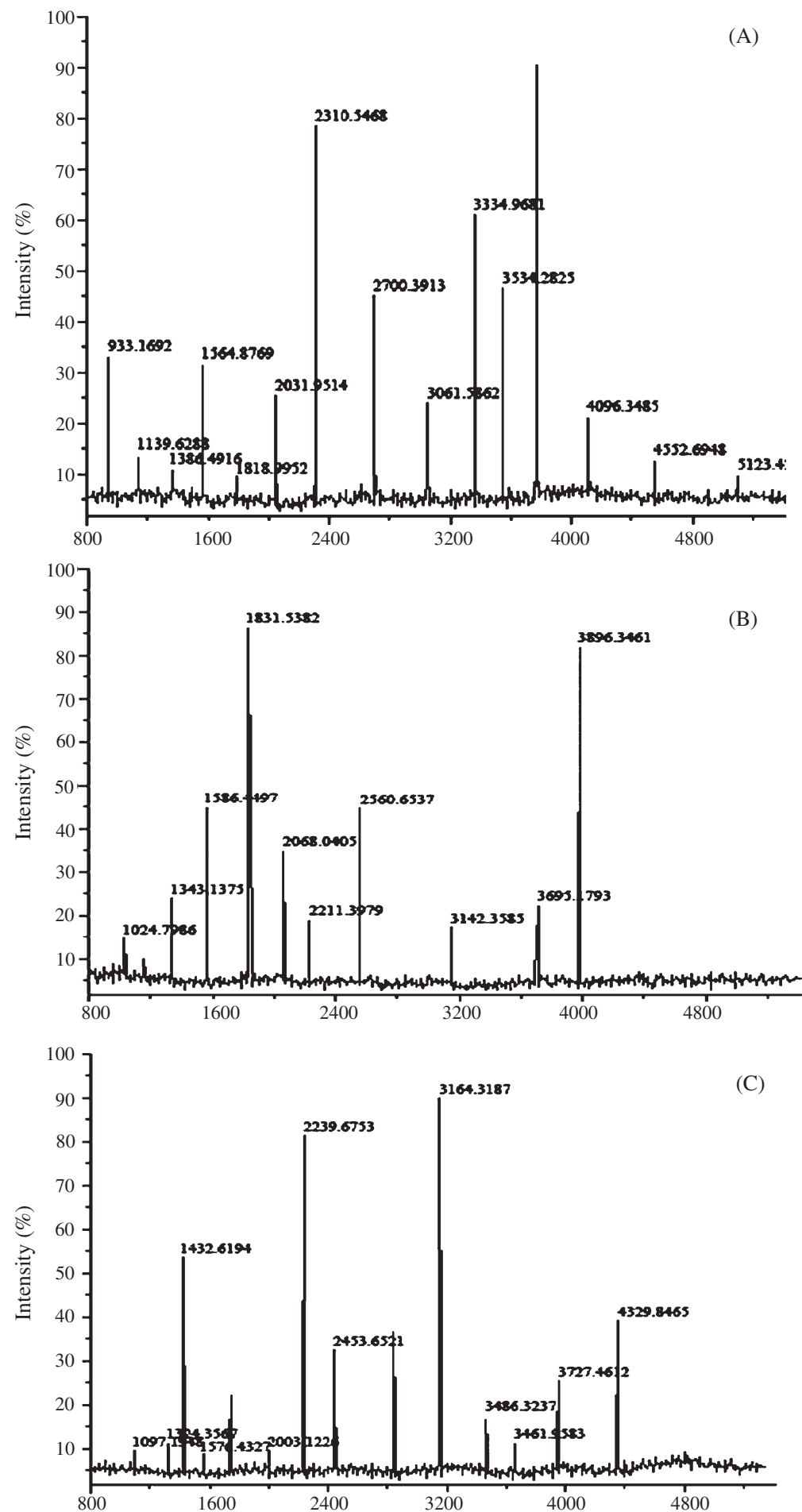

Fig. 5. MALDI-TOF-MS analysis of differential proteins. (A) MALDI-TOF-MS mass spectrum of spot 5 identified as CDKN1 according to the marched peaks was shown. (B) MALDI-TOF-MS mass spectrum of spot 7 identified as Skp2 according to the marched peaks was shown. (C) MALDI-TOF-MS mass spectrum of spot 9 identified as fibronectin according to the marched peaks was shown. 


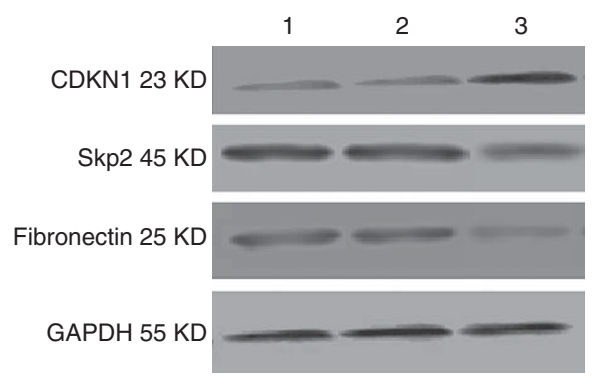

Fig. 6. Representative Western blot analysis of CDKN1, Skp2 and Fibronectin using GAPDH as internal control among SGC-7901, SGC-7901/EV and SGC-7901/let-7a cell lines. Compared with SGC-7901/EV and SGC-7901, SGC-7901/let-7a had obvious upregulation of CDKN1, and a marked down-regulations of Skp2 and Fibronectin 1: SGC-7901; 2: SGC-7901/EV; 3: SGC-7901/let-7a.

results showed that in SGC-7901/let-7a cells, Cyclindependent kinase inhibitor 1 protein level significantly increased, while S-phase kinase-associated protein 2 and Fibronectin were reduced, compared to SGC7901/EV and SGC-7901 cells $(P<0.05)$, as can be seen from the immunoblots shown in Fig. 6. The result verified that these differentially expressed proteins were related to let-7a in gastric carcinoma cells.

\section{Discussion}

This study combined a proteomic approach with gene therapy techniques to identify proteins associated with the function of let-7a in gastric carcinoma. We successfully used gene clone technology constructing the let-7a lentivirus and stably up-regulating let-7a gene expression in SGC-7901 cell line. As a reliable cell model, the well-resolved, reproducible 2-DE patterns of SGC-7901/let-7a, SGC-7901/EV, and SGC-7901 were established. Ten differentially expressed proteins spots among the three cell lines were identified by MALDI-TOF-MS. Antioxidant protein 2, Insulin-like growth factor binding protein 2, Protein disulfide isomerase A2, C-1-tetrahydrofolate synthase, Cyclin-dependent kinase inhibitor1, Rho-GTPase activating protein 4 were upregulated in SGC-7901/let-7a cells, while S-phase kinase-associated protein 2, Platelet membrane glycoprotein, Fibronectin, Cks1 protein were downregulated. Some of the differential expression proteins are known to be associated with the let- $7 \mathrm{a}$ function, and others may be novel proteins associated with let-7a function. These differential proteins could provide new clues to elucidate the mechanisms of let-7a in gastric carcinoma.

CDKN1, a protein that regulates cell cycle progression, terminal differentiation and apoptosis, mediates p53-dependent G1 growth arrest. Earlier studies supported the view that CDKN1 suppresses tumors by promoting cell cycle arrest in response to various stimuli. Additionally, substantial evidence from biochemical and genetic studies indicates that CDKN1 acts as a master effector of multiple tumour suppressor pathways for promoting anti-proliferative activities that are independent of the classical p53 tumor suppressor pathway $[16,17]$. Skp2 is an F-box protein that plays a critical role in coordinating the G1/S transition and progression through the $S$ phase of the mammalian cell cycle $[18,19]$. It is mainly responsible for ubiquitylation and subsequent degradation of cell cycle regulators, and in particular the cell cycle inhibitor p2 $7^{\mathrm{Kip} 1}$. Fibronectin was discovered over thirty years ago as a protein abundantly secreted by tumor cells. Intense investigation of the function of fibronectin in vitro led to the discovery of integrins and other proteins involved cell motility and in the bi-directional communication between cells and their environment. Fibronectin is highly up-regulated around newly developing vasculature during embryogenesis and in pathological conditions including atherosclerosis, cardiac hypertrophy, and tumorigenesis. Recent genetic studies in fish, frogs and mice have suggested that fibronectin functions in maintaining or establishing cell polarity and its splice variants in development of vascular smooth muscle cells [20]. Cks1, a member of the highly conserved Suc1/Cks family of cell cycle regulatory proteins binds to Skp2 and promotes the association of Skp2 with p27 phosphorylated on Thr-187 [21]. The general notion that functional platelets are important for successful hematogenous tumor metastasis had been inaugurated more than 4 decades ago and has since been corroborated in numerous experimental settings. Pivotal preclinical experiments have demonstrated that hematogenous tumor spread was dramatically diminished when platelets were depleted from the circulation [22]. Cell migration affects all morphogenetic processes and contributes to numerous diseases, including cancer. The Rho GTPases regulate adhesion by directly controlling the balance between actin-mediated protrusion and myosin II-mediated contraction [23, 24]. 
Adhesion formation and disassembly drive the migration cycle by activating Rho GTPases, which in turn regulate actin polymerization and myosin II activity, and therefore adhesion dynamics. Insulin-like growth factor binding protein 2 (IGFBP-2) mRNA is already expressed in preimplantation embryos, and expression continues at high levels in many tissues during embryonic and fetal development. In the postnatal period, IGFBP-2 is the second most abundant IGFBP in the circulation and is present in various other biological fluids and tissues of many vertebrate species [25]. IGFBP-2 is overexpressed in many malignancies and is often correlated with an increasingly malignant status of the tumor, pointing to a potential involvement of IGFBP-2 in tumorigenesis. Antioxidant protein 2 (AOP2) is a novel thiol-dependent antioxidant that functions to scavenge particular hydroperoxides in the cell and mediate specific signals. There is also evidence supporting a role for AOP2 in certain disease processes including atherosclerosis. Further evaluation of this protein and its substrate specificity will likely shed light on its precise role in cellular oxidant defense, signal transduction and pathogenesis [26]. Protein disulfide isomerase (PDI) catalyzes protein folding and thiol-disulfide interchange reactions. The enzyme is localized in the lumen of endoplasmic reticulum (ER) and is abundant in secretory cells of various tissues [27]. C-1-tetrahydrofolate synthase was also differentially expressed in different human leukemia and lymphoma cell lines. This provided insights into the molecular basis of these malignancies and its role in cellular properties [28].

Considering the functions of the identified differential proteins, it is reasonable to predict that the proteins such as Cyclin-dependent kinase inhibitor 1, S-phase kinase-associated protein 2 and fibronectin are associated with the function of let-7a in SGC7901. Introducing gene augmentation into proteomics revealed a novel let-7a signal pathway. These findings provide the basis to comprehensively investigate the functional mechanisms of let-7a in gastric carcinoma.

\section{Acknowledgments}

This work was supported by research grants from the key science and technology projects of Guangxi Zhuang autonomous region China (10124001A-49).

The authors declare no competing financial interests.

\section{References}

[1] R.S. Pillai, MicroRNA function: Multiple mechanisms for a tiny RNA? RNA 12 (2005), 1753-1761.

[2] P.D. Zamore and B. Haley, Ribo-gnome: The big word of small RNAs, Science 309 (2005), 1519-1524.

[3] B.P. Lewis, C.B. Burge and D.P. Bartel, Conserved seed pairing,often flanked by adenosines,indicates that thousands of human genes are microRNA targets, Cell 120 (2005), 15-20.

[4] C.M. Croce and G.A. Calin, microRNA, cancer, stem cell division, Cell 122 (2005), 6-7.

[5] C.Z. Chen, L. Li, H.F. Lodish and D.P. Bartel, MicroRNA modulate hematopoietic lineage differentiation, Science $\mathbf{3 0 3}$ (2004), 83-86.

[6] D. Didiano and O. Hobert, Perfect seed pairing is not a generally reliable predictor for microRNA-target interactions, Nat Struct Mol Biol 13 (2006), 849-851.

[7] N.R. Smalheiser and V.I. Torvik, Complications in mammalian microRNA target prediction, Methods Mol Biol 342 (2006), $115-127$.

[8] Y.M. Zhu, Z.X. Zhong and Z.M. Liu, Significance and relationship between let-7a and gastric mucosa cancerization, World $J$ Gastroenterology 16 (2010), 3325-3329.

[9] Y.M. Zhu, Z.X. Zhong and Z.M. Liu, Lentiviral vectormediated upregulation of let-7a inhibits gastriccarcinoma cell growth in vitro and in vivo, Scandinavian Journal of Gastroenterology 46 (2011), 53-59.

[10] Y.M. Zhu, Z.M. Liu and W.B. Bai, Experimental study on the effect of let-7a in Cell cycle of human gastric carcinoma cell line, Chinese Journal of Experimental Surgery 16 (2010), 424.

[11] M.R. Wilkins, J.C. Sanchez and A.A. Gooley, Progress with proteome projects: Why all proteins expressed by a genome should be identified and how to do it, Biotechnol Genet Eng Rev 13 (1996), 19-50.

[12] Y. Sun, H. Yi and P.F. Zhang, Identification of differential proteins in nasopharyngeal carcinoma cells with p53 silence by proteome analysis, FEBS Letters 581 (2007), 131-139.

[13] B. Zhang, P. Metharom and H. Jullie, The significance of controlled conditions in lentiviral vector titration and in the use of multiplicity of infection (MOI) for prediction gene transfer events, Genet Vaccines Ther 2 (2004), 6.

[14] P.S. Bernard and C.T. Wittwer, Real-Time PCR technology for cancer diagnostics, Clin Chem 48 (2002), 1178-1185.

[15] G. Candiano, M. Bruschi and L. Musante, Blue silver: A very sensitive colloidal Coomassie G-250 staining for proteome analysis, Electrophoresis 25 (2004), 1327-1333.

[16] C. Deng, P. Zhang, J.W. Harper, S.J. Elledge and P. Leder, Mice lacking p21CIP1/WAF1 undergo normal development, but are defective in G1 checkpoint control, Cell 82 (1995), 675-684.

[17] I.B. Roninson, Oncogenic functions of tumour suppressor p21Waf1/Cip1/Sdi1: Association with cell senescence and tumour-promoting activities of stromal fibroblasts, Cancer Lett 179 (2002), 1-14.

[18] C. Catzavelos, N. Bhattacharya and Y.C. Ung, Decreased levels of the cell-cycle inhibitor p27Kip1 inhibitor protein: Prognostic implications in primary breast cancer, Nat Med 3 (1997), 227-230. 
[19] V. Esposito, A. Baldi and A. De Luca, Prognostic role of the cyclin dependent kinase inhibitor p27 in non-small lung cancer, Cancer Res 57 (1997), 3382-3385.

[20] S. Astrof and R.O. Hynes, Fibronectins in vascular morphogenesis, Angiogenesis 12 (2009), 165-175.

[21] D. Sitry, M.A. Seeliger and T.K. Ko, Three different binding sites of Cks1 are required for p27-ubiquitin ligation, $J$ Biol Chem 44 (2002), 42233-42240.

[22] L. Erpenbeck and M.P. Schon, Deadly allies: The fatal interplay between platelets and metastasizing cancer cells, Blood 115 (2010), 3427-3436.

[23] A.B. Jaffe and A. Hall, Rho GTPases: Biochemistry and biology, Annu Rev Cell Dev Biol 21 (2005), 247-269.

[24] J.T. Parsons, A.R. Horwitz, M. and A. Schwartz, Cell adhesion: Integrating cytoskeletal dynamics and cellular tension, Nat Rev Mol Cell Biol 11 (2010), 633-643.
[25] A. Hoeflich, R. Reisinger and H. Lahm, Insulin-like growth factor-binding protein 2 in tumorigenesis: Protector or promoter? Cancer Res 61 (2001), 8601-8610.

[26] S.A. Phelan, AOP2 (antioxidant protein 2): Structure and function of a unique thiol-specific antioxidant, Antioxid Redox Signal 1 (1999), 571-584.

[27] M.G. Desilva, J. Lu and G. Donadel, Characterization and chromosomal localization of a new protein disulfide isomerase, PDIp, highly expressed in human pancreas, DNA Cell Biol 15 (1996), 9-16.

[28] S. Gez, B. Crossett and R.I. Christopherson, Differentially expressed cytosolic proteins in human leukemia and lymphoma cell lines correlate with lineages and functions, Biochim Biophys Acta 1774 (2007), 1173-1183. 


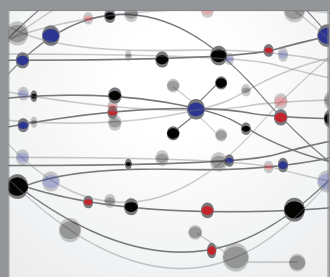

The Scientific World Journal
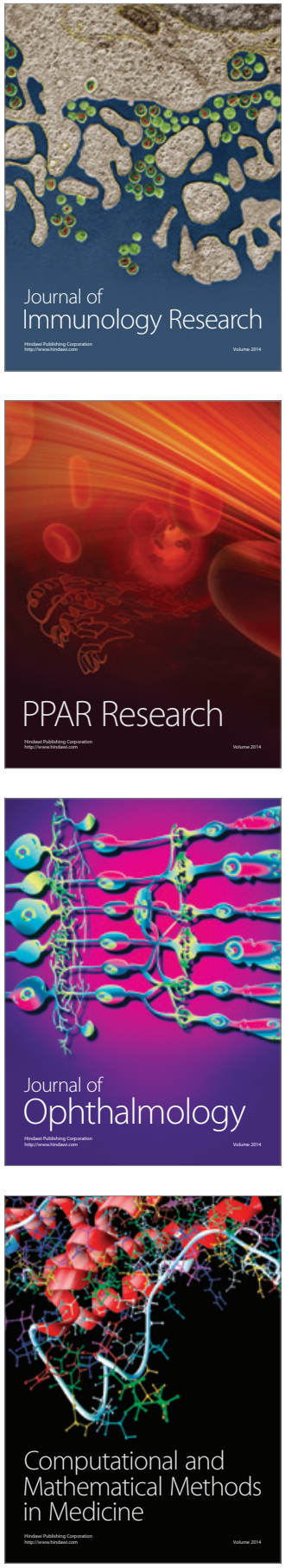

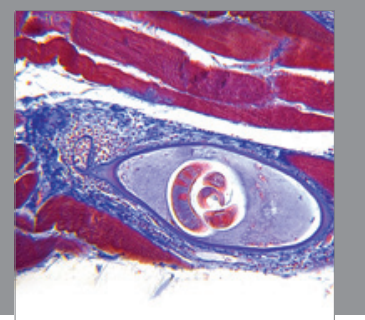

Gastroenterology

Research and Practice
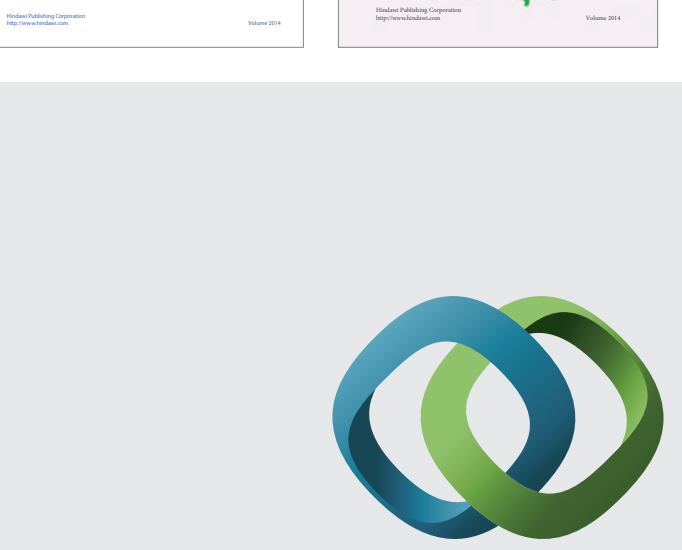

\section{Hindawi}

Submit your manuscripts at

http://www.hindawi.com
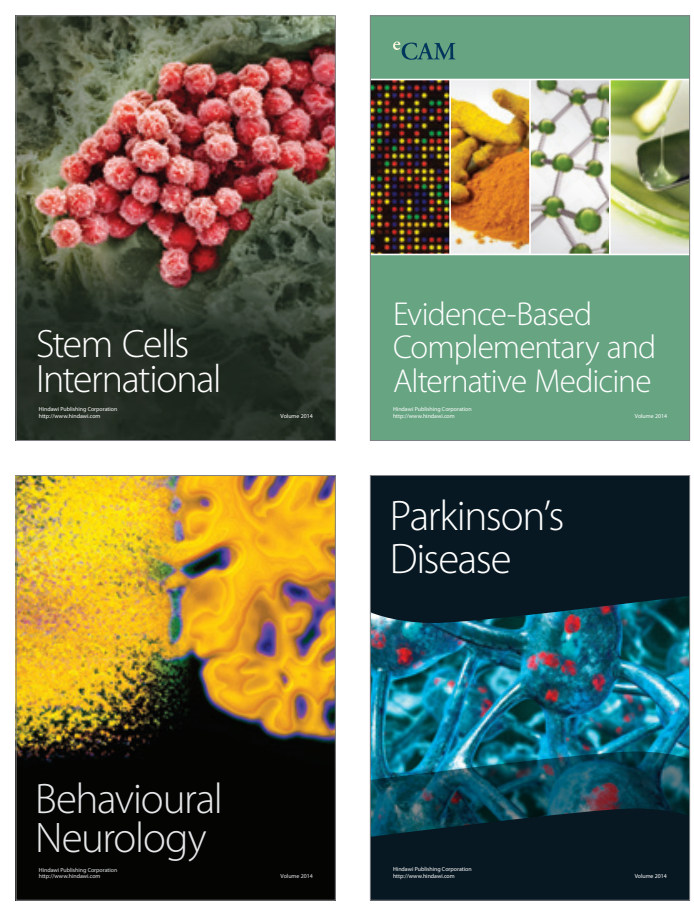

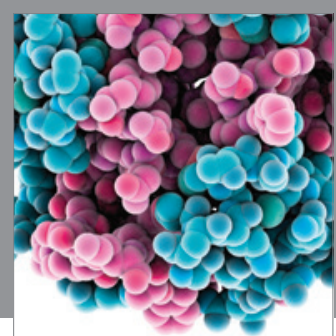

Journal of
Diabetes Research

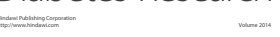

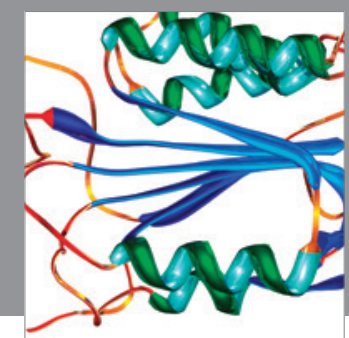

Disease Markers
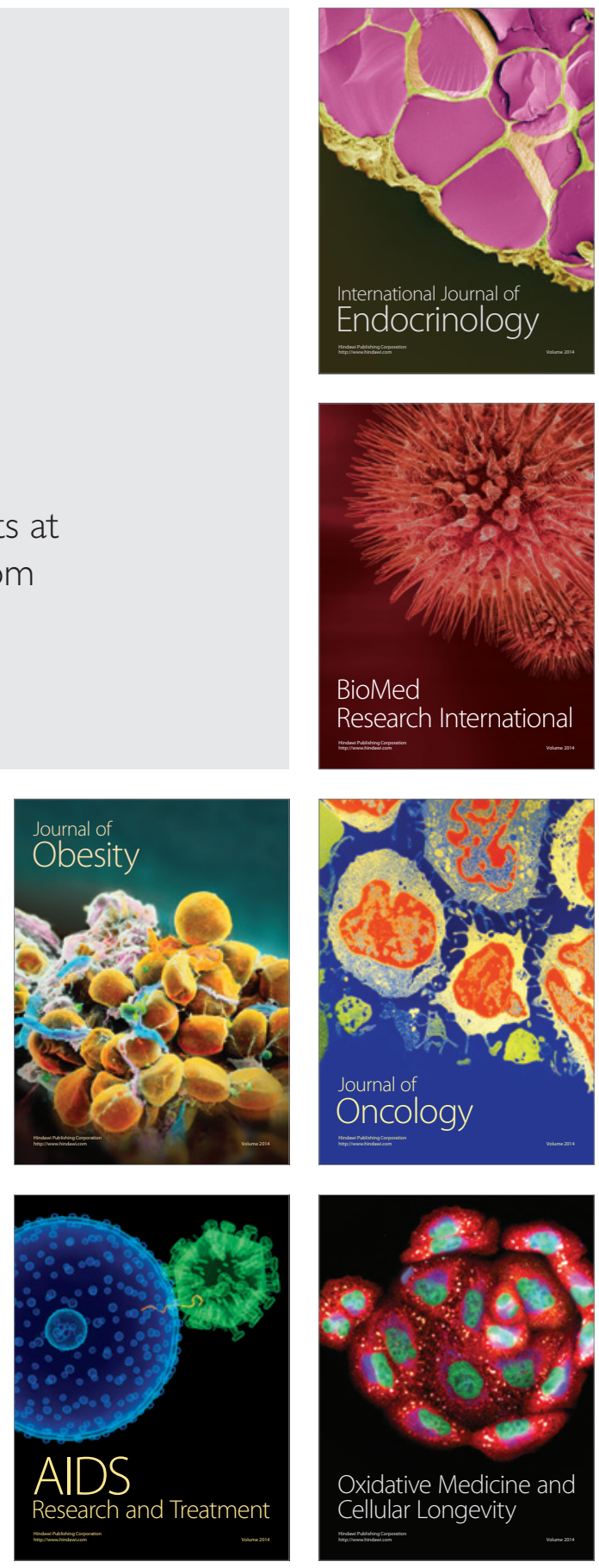